\title{
Large $Q^{2}$ electrodisintegration of the deuteron
}

\author{
Misak M. Sargsian \\ Department of Physics, Florida International University, Miami, FL 33199
}

\begin{abstract}
The break up of the deuteron is studied at high $Q^{2}$ kinematics, with main motivation to probe the deuteron at small internucleon distances. For this, two main issues are studied: electromagnetic interaction of the virtual photon with the bound nucleon and the strong interactions of the produced baryons in the final state of the reaction. Within virtual nucleon approximation we developed a new prescription to account for the bound nucleon effects in the electromagnetic interaction. The final state interaction at high $Q^{2}$ kinematics is calculated within generalized eikonal approximation (GEA). Comparison with the first experimental data confirm GEA's early prediction that the rescattering is maximal at $\sim 70^{0}$ of recoil nucleon production relative to the momentum of the virtual photon. Also the forward recoil nucleon angles are best suited for studies of the deuteron at small distances.
\end{abstract}




\section{INTRODUCTION}

Deuteron represents an ideal micro-lab for studies of the structure of $N N$ interaction ranging from the intermediate to very small distances (see e.g. [1] 3 ].

The simplest reaction which could be used to investigate short-range $N N$ interactions in nuclear targets is the exclusive electrodisintegration of the deuteron in which large magnitudes of the relative momentum of the $\mathrm{pn}$ system in the ground state are probed. Expectations that this can be achieved only at high-momentum transfer reactions [4-7] was confirmed in a series of high energy experiments [8 13] involving various nuclei.

Three experiments 14-16] have been performed recently using deuteron target at relatively high (up to $6 \mathrm{GeV}$ ) energy electron beam of the Jefferson Lab and more comprehensive experimental program will follow after the $12 \mathrm{GeV}$ upgrade of the lab. This makes the development of theoretical approaches for the description of high $Q^{2}$ electro-nuclear processes involving deuteron a pressing issue. Since mid 90's there were an intensive efforts in developing such theoretical approaches [18 27].

In this work [17] we study one particular process that is high $Q^{2}$ disintegration of the deuteron with probing large magnitudes of recoil nucleon momenta. The model is based on virtual nucleon approximation in which the struck nucleon is treated as an off-shell particle. The main theoretical framework is based on the generalized eikonal approximation (GEA) [7, 20, 28, 31] which allows us to represent the reaction through the set of covariant diagrams (Fig.1) for which effective Feynman diagram rules can be defined. Because of the covariance of the diagrams the virtualities involved in the scattering amplitudes are defined unambiguously. This allows us to develop a self consistent approach for accounting for the binding effects in the high $Q^{2}$ electromagnetic interaction off the bound nucleon. The second important feature of GEA is that final-state interaction of produced two nucleons is calculated without requiring stationary approximation for the recoil nucleons - this is important feature for calculating processes in which the recoil nucleon is produced with large momenta.

\section{MAIN ASSUMPTIONS OF VIRTUAL NUCLEON APPROXIMATION}

First, one considers only the $p n$ component of the deuteron, neglecting inelastic initial state transitions. Since the deuteron is in a isosinglet state this will correspond to restricting the kinetic energy of recoil nucleon to

$$
T_{N}<2\left(m_{\Delta}-m_{N}\right) \sim\left(m_{N^{*}}-m\right) \sim 500 \mathrm{MeV}
$$

where $m, m_{\Delta}$ and $m_{N^{*}}$ are masses of the nucleon and low-lying non-strange baryonic resonances.

Then, one neglects by the negative energy projection of the virtual nucleon propagator. This can be justified if,

$$
M_{d}-\sqrt{m^{2}+p^{2}}>0,
$$

where $M_{d}$ is the mass of the deuteron and $p$ is the relative momentum of the bound $p n$ system.

The third assumption which is made in the calculation is that at large $Q^{2}\left(>1 \mathrm{GeV}^{2}\right)$ the interaction of virtual photon with exchanged mesons are a small correction and can be neglected (see e.g. discussions in Ref. [4, 7]). 

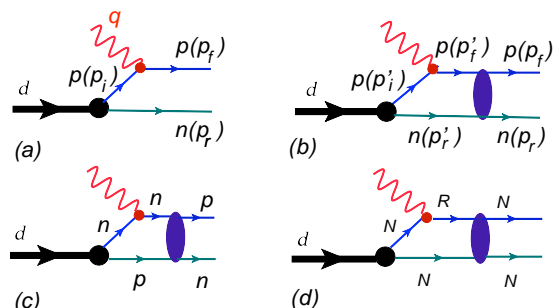

FIG. 1: GEA diagrams

\section{GENERALIZED EIKONAL APPROXIMATION}

The assumptions discussed above allow us to restrict the consideration by the set of Feynman diagrams presented in Fig.(1), which can be calculated based on the effective Feynman diagram rules derived in GEA [7]. This work does not include calculation of the diagram of Fig.1d which is currently in progress.

\section{Plane Wave Impulse Approximation Amplitude:}

Starting with the covariant form of the amplitude of Fig1.a, we calculate explicitly the off shell part of the electromagnetic amplitude through the one-shell positive energy reduction of the propagator of the spectator nucleon[17]. This procedure results to the PWIA amplitude[17]:

$$
\left\langle s_{f}, s_{r}\left|A_{0}^{\mu}\right| s_{d}\right\rangle=\sqrt{2} \sqrt{(2 \pi)^{3} 2 E_{r}} \sum_{s_{i}} J_{N}^{\mu}\left(s_{f}, p_{f} ; s_{i}, p_{i}\right) \Psi_{d}^{s_{d}}\left(s_{i}, p_{i}, s_{r}, p_{r}\right),
$$

with

$$
J_{N}^{\mu}\left(s_{f}, p_{f} ; s_{i}, p_{i}\right)=J_{N, o n}^{\mu}\left(s_{f}, p_{f} ; s_{i}, p_{i}\right)+J_{N, o f f}^{\mu}\left(s_{f}, p_{f} ; s_{i}, p_{i}\right) .
$$

where $J_{N, \text { on }}^{\mu}$ is the covariant on-shell electromagnetic current of the nucleon and

$$
J_{N, o f f}^{\mu}\left(s_{f}, p_{f} ; s_{i}, p_{i}\right)=\bar{u}\left(p_{f}, s_{f}\right) \Gamma_{\gamma^{*} N}^{\mu} \gamma^{0} u\left(p_{i}, s_{i}\right) \frac{E_{i}^{o f f}-E_{i}^{o n}}{2 m},
$$

in which $E_{i}^{o f f}=M_{d}-E_{i}^{o n}$ and $E_{i}^{o n}=\sqrt{m^{2}+p_{i}^{2}}, \vec{p}_{i}=-\vec{p}_{r}$. Note that the total current in Eq.(4) is conserved since it is derived from the gauge invariant amplitude and no additional conditions are needed to restore the current conservation.

\section{Forward Elastic and Charge Interchange Final State Interaction Amplitudes:}

Applying the effective Feynman diagram rules to the diagrams of Fig.(1)b,c and projecting (similar to PWIA) the propagator of the spectator nucleon to its positive energy solution for the forward rescattering amplitude we obtain:

$$
\begin{aligned}
& \left\langle s_{f}, s_{r}\left|A_{1}^{\mu}\right| s_{d}\right\rangle=\frac{i \sqrt{2}(2 \pi)^{\frac{3}{2}}}{4} \sum_{s_{f}^{\prime}, s_{r}^{\prime}, s_{i}} \int \frac{d^{2} p_{r}^{\prime}}{(2 \pi)^{2}} \frac{\sqrt{2 \tilde{E}_{r}^{\prime}} \sqrt{s\left(s-4 m^{2}\right)}}{2 \tilde{E}_{r}^{\prime}|q|} \times \\
& \left\langle p_{f}, s_{f} ; p_{r}, s_{r}\left|f^{N N, o n}(t, s)\right| \tilde{p}_{r}^{\prime}, s_{r}^{\prime} ; \tilde{p}_{f}^{\prime}, s_{f}^{\prime}\right\rangle \cdot J_{N}^{\mu}\left(s_{f}^{\prime}, p_{f}^{\prime} ; s_{i}, \tilde{p}_{i}^{\prime}\right) \cdot \Psi_{d}^{s_{d}}\left(s_{i}, \tilde{p}_{i}^{\prime}, s_{r}^{\prime}, \tilde{p}_{r}^{\prime}\right) \\
& -\frac{\sqrt{2}(2 \pi)^{\frac{3}{2}}}{2} \sum_{s_{f}^{\prime}, s_{r}^{\prime}, s_{i}} \mathcal{P} \int \frac{d p_{r, z}^{\prime}}{2 \pi} \int \frac{d^{2} p_{r}^{\prime}}{(2 \pi)^{2}} \frac{\sqrt{2 E_{r}^{\prime}} \sqrt{s\left(s-4 m^{2}\right)}}{2 E_{r}^{\prime}|\mathbf{q}|} \times
\end{aligned}
$$




$$
\frac{\left\langle p_{f}, s_{f} ; p_{r}, s_{r}\left|f^{N N, o f f}(t, s)\right| p_{r}^{\prime}, s_{r}^{\prime} ; p_{f}^{\prime}, s_{f}^{\prime}\right\rangle}{p_{r, z}^{\prime}-\tilde{p}_{r, z}^{\prime}} J_{N}^{\mu}\left(s_{f}^{\prime}, p_{f}^{\prime} ; s_{i}, p_{i}^{\prime}\right) \cdot \Psi_{d}^{s_{d}}\left(s_{i}, p_{i}^{\prime}, s_{r}^{\prime}, p_{r}^{\prime}\right)
$$

where $\tilde{p}_{r}^{\prime}=\left(p_{r, z}-\Delta, p_{r, \perp}^{\prime}\right), \tilde{E}_{r}^{\prime}=\sqrt{m^{2}+\tilde{p}_{r}^{\prime 2}}, \tilde{p}_{i}^{\prime}=p_{d}-\tilde{p}_{r}^{\prime}$ and $\tilde{p}_{f}^{\prime}=\tilde{p}_{i}^{\prime}+q$.

In high energy limit in which the helicity conservation of small angle NN scattering is rather well established the on-shell amplitude is predominantly imaginary and can be parameterized in the form

$$
\left\langle p_{f}, s_{f} ; p_{r}, s_{r}\left|f^{N N, o n}(t, s)\right| \tilde{p}_{r}^{\prime}, s_{r}^{\prime} ; \tilde{p}_{f}^{\prime}, s_{f}^{\prime}\right\rangle=\sigma_{t o t}^{p n}(i+\alpha) e^{\frac{B}{2} t} \delta_{s_{f}, s_{f}^{\prime}} \delta_{s_{r}, s_{r}^{\prime}},
$$

where $\sigma_{t o t}^{p n}(s), B(s)$ and $\alpha(s)$ are found from fitting of experimental data on elastic $p n \rightarrow$ $p n$ scattering. For the half-off-shell part of the $f^{N N, o f f}$ amplitude we use the following parameterization:

$$
f^{N N, o f f}=f^{N N, o n} e^{B\left(m_{o f f}^{2}-m^{2}\right)}
$$

where $m_{o f f}^{2}=\left(M_{d}-E_{r}^{\prime}+q_{0}\right)^{2}-\left(p_{r}^{\prime}+q\right)^{2}$.

For the charge-exchange final state interaction amplitude (Fig.1c) the derivation is similar and it is expressed through the charge-exchange $p n \rightarrow n p$ scattering amplitude.
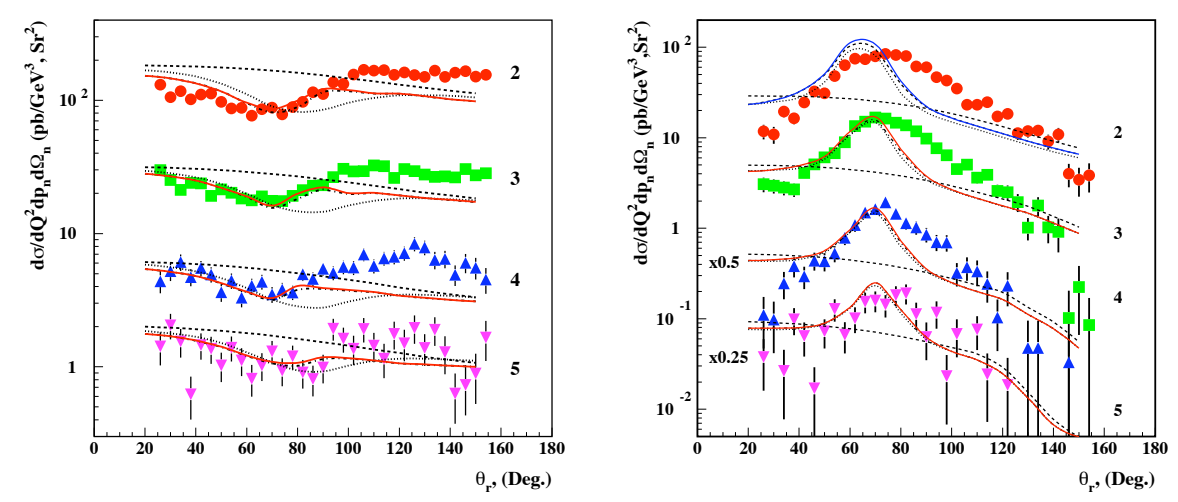

FIG. 2: Dependence of the differential cross section on the direction of the recoil neutron momentum. The data are from Ref.[15]. Dashed line - PWIA calculation, dotted line - PWIA+ pole term of forward FSI, dash-dotted line - PWIA + forward FSI, solid line - PWIA + forward and charge exchange FSI. The momenta of the recoil neutron in (a) and (b) are restricted to $200<p_{r}<300 \mathrm{MeV} / \mathrm{c}$ and $400<p_{r}<600 \mathrm{MeV} / \mathrm{c}$ respectively. The labels $2,3,4$ and 5 correspond to the following values of $Q^{2}=2 \pm 0.25 ; 3 \pm 0.5 ; 4 \pm 0.5 ; 5 \pm 0.5 \mathrm{GeV}^{2}$. The data sets and calculations for " 4 " and " 5 " in (b) are multiplied by 0.5 and 0.25 respectively

\section{Deuteron Wave Function:}

To fix the normalization of the deuteron wave function we use the fact that the deuteron elastic charge form-factor $G_{C} \rightarrow 1$ at $Q^{2} \rightarrow 0$. Using above condition in the elastic deuteron scattering amplitude one can relate the deuteron wave function in virtual nucleon approximation to the nonrelativistic deuteron wave function as follows[17]

$$
\Psi_{d}(p)=\Psi_{d}^{N R}(p) \frac{M_{d}}{2\left(M_{d}-\sqrt{m^{2}+p^{2}}\right)} .
$$


This relation provides a smooth transition to the nonrelativistic wave function $\Psi^{N R}$ in the small momentum limit.

\section{COMPARISON WITH EXPERIMENTAL DATA AND SOME CONCLU- SIONS}

In the last few years three experiments [14-16] produced the first data at relatively large $Q^{2}$ kinematics. The experiment of Ref.[15]) covered the highest to date $Q^{2}$ range from $2-5 \mathrm{GeV}^{2}$. Comparison with these experimental data are given in Fig 2 which allow us to make the following conclusions:

- the angular distribution clearly exhibits an eikonal feature, with the minimum (Fig,2(a)) or maximum (Fig.2(b)) at transverse kinematics due to the final state interaction. The most important result is that the maximum of FSI is at recoil angles of $70^{\circ}$ in agreement with the GEA prediction of Ref.[28]. Note that the conventional Glauber theory predicted $90^{\circ}$ for the FSI maximum.

- The disagreement of the calculation with the data at $\theta_{r}>70^{0}$ appears to be due to the isobar contribution at the intermediate state of the reaction. This region corresponds to $x<1$ and it is kinematically closer to the threshold of $\Delta$-isobar electroproduction. The comparisons also indicate that the relative strength of the $\Delta$-isobar contribution diminishes with an increase of $Q^{2}$ and at neutron production angles $\theta_{r} \rightarrow 180^{\circ}$.

- The forward direction of the recoil nucleon momentum, being far from the $\Delta$-isobar threshold, exhibits a relatively small contribution due to FSI. This indicates that the forward recoil angle region is best suited for studies of PWIA properties of the reaction such as the off-shell electromagnetic current and deuteron wave function.

These comparisons clearly show that the forward angles of spectator nucleon production is best suited for isolating PWIA scattering off the virtual nucleon. Therefore this kinematic region provides the most optimal condition for probe and studying the $N N$ interaction at short internucleon separations.

\section{Acknowledgments:}

This work is supported by the U.S. Department of Energy Grant under Contract DE-FG0201ER41172.

[1] R. A. Gilman and F. Gross, J. Phys. G 28, R37 (2002).

[2] L. L. Frankfurt, G. A. Miller, M. M. Sargsian and M. I. Strikman, Phys. Rev. Lett. 84, 3045 (2000).

[3] C. G. Granados and M. M. Sargsian, arXiv:1007.4705 [hep-ph].

[4] M. M. Sargsian et al., J. Phys. G 29, R1 (2003).

[5] L. Frankfurt, M. Sargsian and M. Strikman, Int. J. Mod. Phys. A 23, 2991 (2008).

[6] L. L. Frankfurt and M. I. Strikman, Phys. Rept. 76, 215 (1981); Phys. Rept. 160, 235 (1988).

[7] M. M. Sargsian, Int. J. Mod. Phys. E 10, 405 (2001).

[8] L. L. Frankfurt, M. I. Strikman, D. B. Day and M. Sargsian, Phys. Rev. C 48, 2451 (1993).

[9] K. Sh. Egiyan et al. [CLAS Collaboration], Phys. Rev. C 68, 014313 (2003).

[10] K. Sh. Egiyan et al. [CLAS Collaboration],Phys. Rev. Lett. 96, 082501 (2006). 
[11] E. Piasetzky, M. Sargsian, L. Frankfurt, M. Strikman and J. W. Watson, Phys. Rev. Lett. 97, 162504 (2006).

[12] R. Shneor et al. [Jefferson Lab Hall A Collaboration], Phys. Rev. Lett. 99, 072501 (2007).

[13] R. Subedi et al., Science 320, 1476 (2008).

[14] P. E. Ulmer et al., Phys. Rev. Lett. 89, 062301 (2002).

[15] K. Sh. Egiyan et al. [the CLAS Collaboration], Phys. Rev. Lett. 98, 262502 (2007).

[16] W. U. Boeglin, Eur. Phys. J. A 24, 77 (2005); L. Coman, “ ${ }^{2} H\left(e, e^{\prime} p\right)$ Studies of the Deuteron at High $Q^{2} "$, PhD. Thesis (unpublished), (2008).

[17] M. M. Sargsian, Phys. Rev. C 82, 014612 (2010).

[18] L. Frankfurt, E. Piasetsky, M. Sargsian and M. Strikman, Phys. Rev. C 51, 890 (1995).

[19] A. Bianconi, S. Jeschonnek, N. N. Nikolaev and B. G. Zakharov, Phys. Lett. B 343, 13 (1995).

[20] L. L. Frankfurt, W. R. Greenberg, G. A. Miller, M. M. Sargsian and M. I. Strikman, Z. Phys. A 352, 97 (1995).

[21] L. L. Frankfurt, E. Piasetzky, M. M. Sargsian and M. I. Strikman,Phys. Rev. C 56, 2752 (1997).

[22] S. Jeschonnek, Phys. Rev. C 63, 034609 (2001).

[23] C. Ciofi degli Atti, L. P. Kaptari and D. Treleani, Phys. Rev. C 63, 044601 (2001).

[24] J. M. Laget, Phys. Lett. B 609, 49 (2005).

[25] S. Jeschonnek and J. W. Van Orden, Phys. Rev. C 78, 014007 (2008); Phys. Rev. C 80, 054001 (2009).

[26] S. Jeschonnek and J. W. Van Orden, Phys. Rev. C 81, 014008 (2010).

[27] J.J. Adam, F. Gross, S. Jeschonnek, P. Ulmer and J. W. Van Orden, Phys. Rev. C 66, 044003 (2002).

[28] L. L. Frankfurt, M. M. Sargsian and M. I. Strikman, Phys. Rev. C 56, 1124 (1997).

[29] M. M. Sargsian, T. V. Abrahamyan, M. I. Strikman and L. L. Frankfurt, Phys. Rev. C 71, 044614 (2005).

[30] C. Ciofi delgi Atti and L. P. Kaptari,Phys. Rev. Lett. 100, 122301 (2008).

[31] M. Alvioli, C. Ciofi degli Atti and L. P. Kaptari, Phys. Rev. C 81, 021001 (2010) 